\title{
The neutron texture diffractometer at the China Advanced Research
}

\section{Reactor}

\author{
LI MeiJuan ${ }^{1}$, LIU XiaoLong ${ }^{1}$, LIU YunTao ${ }^{1}$, TIAN GengFang ${ }^{1}$, GAO JianBo ${ }^{1}$, \\ YU ZhouXiang ${ }^{1}$, LI YuQing ${ }^{1}$, WU LiQi ${ }^{1}$, YANG LinFeng ${ }^{1}$, SUN Kai ${ }^{1}$, \\ WANG HongLi ${ }^{1}$, J.R. Santisteban ${ }^{2}$, CHEN DongFeng ${ }^{1 *}$ \\ 1. Department of Nuclear Physics, China Institute of Atomic Energy, Beijing, 102413, China \\ 2. Centro Atómico Bariloche, CNEA, Bariloche, 8400, Argentina
}

\begin{abstract}
The first neutron texture diffractometer in China has been built at the China Advanced Research Reactor, due to strong demand for texture measurement with neutrons from the domestic user community. This neutron texture diffractometer has high neutron intensity, moderate resolution and is mainly applied to study texture in commonly used industrial materials and engineering components. In this paper, the design and characteristics of this instrument are described. The results for calibration with neutrons and quantitative texture analysis of Zirconium alloy plate are presented. The comparison of texture measurements with the results obtained in HIPPO at LANSCE and Kowari at ANSTO illustrates the reliability of the texture diffractometer.
\end{abstract}

Key words: Neutron diffraction, neutron texture diffractometer, bulk texture, industrial application

PACS:

\section{Introduction}

Usually, the orientations of crystallites in polycrystalline materials have some preferred direction. The structure with this preferred orientation is known as the texture of the polycrystalline material ${ }^{[1]}$. As an intrinsic feature of metals, ceramics and polymers ${ }^{[2]}$, texture strongly affects many properties of polycrystalline materials ${ }^{[3]}$, and is seen as one of the most important features to fully characterize any kind of polycrystalline material at the microstructural level. Usually, the texture distribution can be quantitatively described by the orientation distribution function (ODF) with respect to a macroscopic sample coordinate system.

Texture determination is usually based on the pole figure measurements by X-ray, synchrotron radiation, electron back-scatter diffraction and neutron diffraction. Among these techniques, neutron diffraction has its own advantages due to the low absorption coefficient of neutrons ${ }^{[3,4]}$ (the penetration depth for most materials in neutron diffraction is a factor of $10^{2}-10^{3}$ larger than for X-ray diffraction).These advantages include: 
1) Large samples can be used, so high accuracy and good statistics as well as bulk texture can be obtained;

2) Accurate determinations of texture can be achieved for coarse-grained, inhomogeneous textured, multiphase samples and for samples with a small volume fraction of second phase;

3) The scattering length of neutron changes irregularly with atomic number, even for various isotopes of the same element, so the texture studies of light element phases, especially in the presence of other phases containing heavy elements, is much easier;

4) Neutrons can be scattered magnetically - this effect is used for magnetic texture analyses;

5) More importantly, texture measurements for samples in non- ambient environments such as high temperature and load can be easily carried out.

Among the texture analysis methods, "Texture analysis by neutron diffraction has become a standard method to investigate bulk textures of various materials" ${ }^{[5]}$. In consequence, more than ten neutron texture diffractometers have been built in different neutron scattering laboratories around the world.

However, like other neutron scattering experiments, pole figure measurement by neutrons requires an intense neutron source such as a nuclear reactor with at least medium neutron flux, or a spallation neutron source. The China Advanced Research Reactor (CARR) at the China Institute of Atomic Energy (CIAE), with a maximum thermal neutron flux of about $8 \times 10^{14} / \mathrm{sec} \cdot \mathrm{cm}^{2[6-7]}$, is well qualified for neutron texture measurements and other neutron scattering experiments. A number of different types of neutron scattering instruments have now been or are being built around the reactor. The Neutron Texture Diffractometer (NTD) is one of these instruments and the first platform for neutron texture measurement in China. In order to make full use of this instrument by the international and domestic user community, the design, characteristics and performance of the newly constructed NTD are introduced in this paper.

\section{Instrument design and characteristics}

The NTD is located at beam tubeH2-1of CARR. Its main components and layout are shown in Figure 1. 


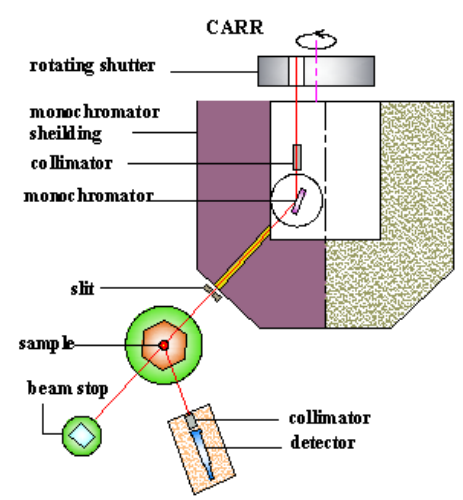

Figure 1 Layout of Neutron Texture Diffractometer at CARR

The design was developed based on the considerations of neutron intensity and instrument resolution. Since pole figure measurements are very time-consuming, the most important factor for a texture diffractometer is to have high neutron intensity. On the other hand, high resolution means that more well-separated reflection peaks can be chosen and samples with larger cells and more complicated structures can be investigated ${ }^{[8-9]}$.

According to the Caglioti, the instrument resolution function as expressed by $A_{1 / 2}$, the full width at half maximum for the peaks, and the integrated peak intensity Lof the instrumentare given as follows [10-12].

$$
\begin{gathered}
A_{1 / 2}^{2}=U \tan ^{2} \theta+V \tan \theta+W \\
U=\frac{4\left(\alpha_{1}^{2} \alpha_{2}^{2}+\alpha_{1}^{2} \beta^{2}+\alpha_{2}^{2} \beta^{2}\right)}{\tan ^{2} \theta_{M}\left(\alpha_{1}^{2}+\alpha_{2}^{2}+4 \beta^{2}\right)} \\
V=\frac{-4 \alpha_{2}^{2}\left(\alpha_{1}^{2}+2 \beta^{2}\right)}{\tan \theta_{M}\left(\alpha_{1}^{2}+\alpha_{2}^{2}+4 \beta^{2}\right)} \\
W=\frac{\alpha_{1}^{2} \alpha_{2}^{2}+\alpha_{1}^{2} \alpha_{3}^{2}+\alpha_{2}^{2} \alpha_{3}^{2}+4 \beta^{2}\left(\alpha_{2}^{2}+\alpha_{3}^{2}\right)}{\alpha_{1}^{2}+\alpha_{2}^{2}+4 \beta^{2}},(1) \\
\mathrm{L}=\frac{\alpha_{1} \alpha_{2} \alpha_{3} \beta}{\left(\alpha_{1}^{2}+\alpha_{2}^{2}+4 \beta^{2}\right)^{1 / 2}},(2)
\end{gathered}
$$

Where $\alpha_{1}, \alpha_{2}$ and $\alpha_{3}$ are the horizontal divergences of the primary, second and third collimators, respectively, $\beta$ is the monochromator mosaic spread, and $2 \theta_{M}$ is the take-off angle of the monochromator.

As can be seen, the above two requirements are in conflict with each other. The instrument resolution can be easily improved by using collimators with smaller horizontal divergence, but this will drastically decrease the diffractometer intensity. It is not possible for a texture diffractometer to achieve both resolution and intensity as high as desired.

If the instrument resolution function is designed higher than that required for the samples to be 
measured, a meaningless loss in diffraction intensity will be caused, which is unacceptable due to the fact that the reactor sources available in the world are not strong enough for neutron scattering experiments. Therefore a compromise has to be made. In the case of NTD at CARR, most of the materials to be measured have simple structures and relatively small cells. For the pole figure measurements of these materials, the instrument is required to have only moderate resolution, which enables it to achieve high neutron intensity. Obviously, in order to make the instrument reasonable and efficient, the best solution is to let the instrument resolution function just match the required one in a large enough $2 \theta$ range.

To obtain the required resolution function, the expression for the separation between adjacent peaks for cubic cells deduced from the Bragg equation was used appropriately in the design (see Equation (3)).Here, $\lambda$ is the neutron wavelength, $2 \theta$ is the scattering angle and $a$ is the size of the unit cell. It is clear that the required resolution function is symmetrical about the minimum $2 \theta=90^{\circ}$.

$$
\Delta(2 \theta)=2 \Delta \theta=\left(\frac{\lambda}{2 a}\right)^{2} \frac{2}{\sin 2 \theta}(3)
$$

In practice, the monochromator was selected before the "matching" procedure so that the parameters $\lambda, 2 \theta_{M}$ and $\beta$ for the instrument were determined first. A single crystal used as monochromator should have high neutron reflectivity, appropriate mosaic spread $\beta$ for high neutron intensity and a proper plane spacing $d$ for the take-off angle $2 \theta_{M}$ and the reflected neutron wavelength $\lambda$ required by a specific spectrometer.

Unfortunately, one has only a few choices since the growth of single crystals large enough for use is very difficult. For our texture diffractometer, a $\mathrm{Cu}(111)$ single crystal from the previous Four Circle Diffractometer (FCD) at the Juelich Center for Neutron Science(JCNS) is used as the monochromator. Single crystals of $\mathrm{Cu}$ have relatively high neutron reflectivity and are often used as monochromators in neutron scattering instruments, but the mosaic spread $\beta$ of this $\mathrm{Cu}(111)$ crystal is only about $6^{\prime}$, which is too small for a texture instrument from the point of view of intensity, although the lower $\beta$ value benefits the resolution for angles below the minimum $\mathrm{A}_{1 / 2}$ position. Fortunately, it is a vertically bent monochromator, so the intensity gain factor of 2-3 can compensate the intensity loss due to the too small $\beta$. This instrument will be equipped with a double focusing HOPG (002) or $\mathrm{Si}(311)$ monochromator in the future.

With the known inter planar spacing of $\mathrm{Cu}(111), \mathrm{d}=2.0871 \AA$, the neutron wavelength $\lambda$ can be 
obtained from the Bragg equation $2 d \sin \theta_{M}=\lambda$. Usually, the take-off angle $2 \theta_{M}$ is set to about $2 \theta=90^{\circ}$, or even a larger angle, so that a good match between the instrument and required resolution functions can be obtained over a larger $2 \theta$ angle range. However, for our instrument the maximum $2 \theta_{M}$ we can choose is restricted to about $2 \theta_{\mathrm{M}}=40^{\circ}$ due to the limited geometric space, and thus the corresponding neutron wavelength becomes $\lambda \approx 1.43 \AA$.Although the use of this low take-off angle will lower the resolution of the instrument, it is still acceptable for most texture measurements, whereas the corresponding neutron wave length $\lambda$ is near the maximum of the incident neutron wave length spectrum, and thus is beneficial to the intensity ${ }^{[13-14]}$.

With the given $\lambda, \beta$ and $2 \theta_{M}$, the range of the required resolution functions was estimated through calculating the required resolution functions for several typical metals and then a series of calculations were carried out for the instrument resolution function for different combinations of $\alpha_{1}, \alpha_{2}$ and $\alpha_{3}$.

Usually, in order to obtain high neutron intensity with relatively small sacrifice of resolution, a large angle $\alpha_{2}$ is chosen. In our case, the second collimator is needless since the natural collimation caused by the geometric layout of the instrument of $\sim 30^{\prime}$ can fully replace the use of $\alpha_{2}$. Therefore, all the calculations for the instrument resolution function were performed with $\alpha_{2}$ equal to $30^{\prime}$. By careful comparisons between the calculated instrument and required resolution functions, three different combinations of $\alpha_{1}, \alpha_{3}$ were finally adopted to meet the requirements of different materials. The results are listed in Table1 and have been used in the construction of the texture diffractometer, and the corresponding instrument resolution functions are plotted in Figure 2.

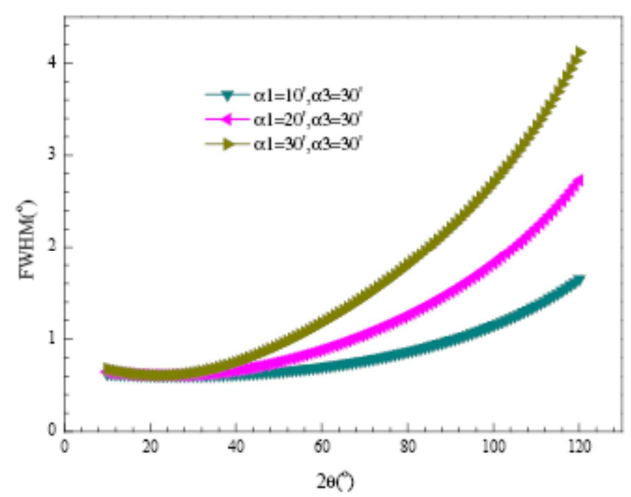

Figure 2Instrument resolution functions for different combinations of $\alpha_{1}$ and $\alpha_{3}$

Besides the monchromator and collimators, the sample table is another important component of the texture diffractometer, although it does not affect the intensity and resolution directly. For our instrument, the four-circle mechanical device of the above-mentioned Juelich FCD was modified and reused as the sample table. With a four-circle device, the pole figure $\mathrm{P}_{\mathrm{hl}}(\alpha, \beta)$ for a specified plane (hkl) 
can be obtained by rotation of the sample through the $\chi\left(0^{\circ} \sim 90^{\circ}\right)$ and $\varphi\left(0^{\circ} \sim 360^{\circ}\right)$ axes with a certain step size to align the various sample orientations along the (hkl) scattering vector, which is usually the bisector of the incident and the diffracted beam, and to record the diffracted intensities at each step using a detector set at the $2 \theta$ position of the (hkl) reflection.

For the pole figure measurement to be realized, the hardware and software for motion control and data acquisition were specially designed by Xiaolong Liu et al. ${ }^{[15]}$.Shown in Figure 3 is the hardware structure. The software was written in the Python language under the Linux system, and has been proved to be reliable by practical pole figure measurements

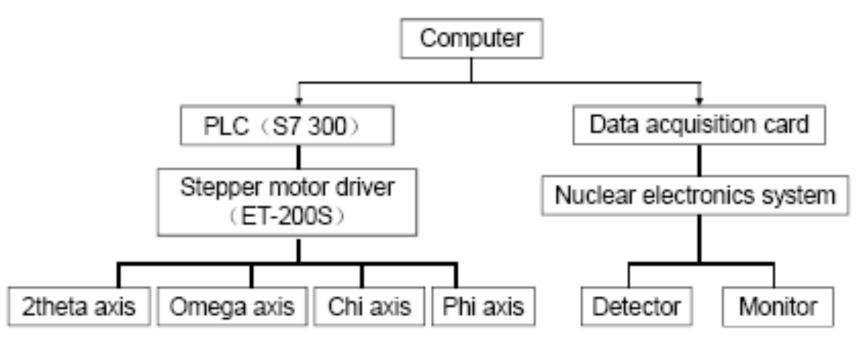

Figure 3Hardware structure of motion control and data acquisition

It is worth mentioning that the ${ }^{3} \mathrm{He}$ single detector used at present will be replaced in the near future by a $200 \mathrm{~mm} \times 200 \mathrm{~mm}$ two-dimensional position sensitive detector (PSD) funded by the International Atomic Energy Agency. With this PSD, it is possible to measure several pole figures simultaneously. Moreover, overlapping pole figures may be separated, which is especially important for materials with line-rich diffraction patterns, such as intermetallic phases, ceramics and composites. The characteristics of this instrument are given in Table1.

Table 1Characteristics of NTD at CARR

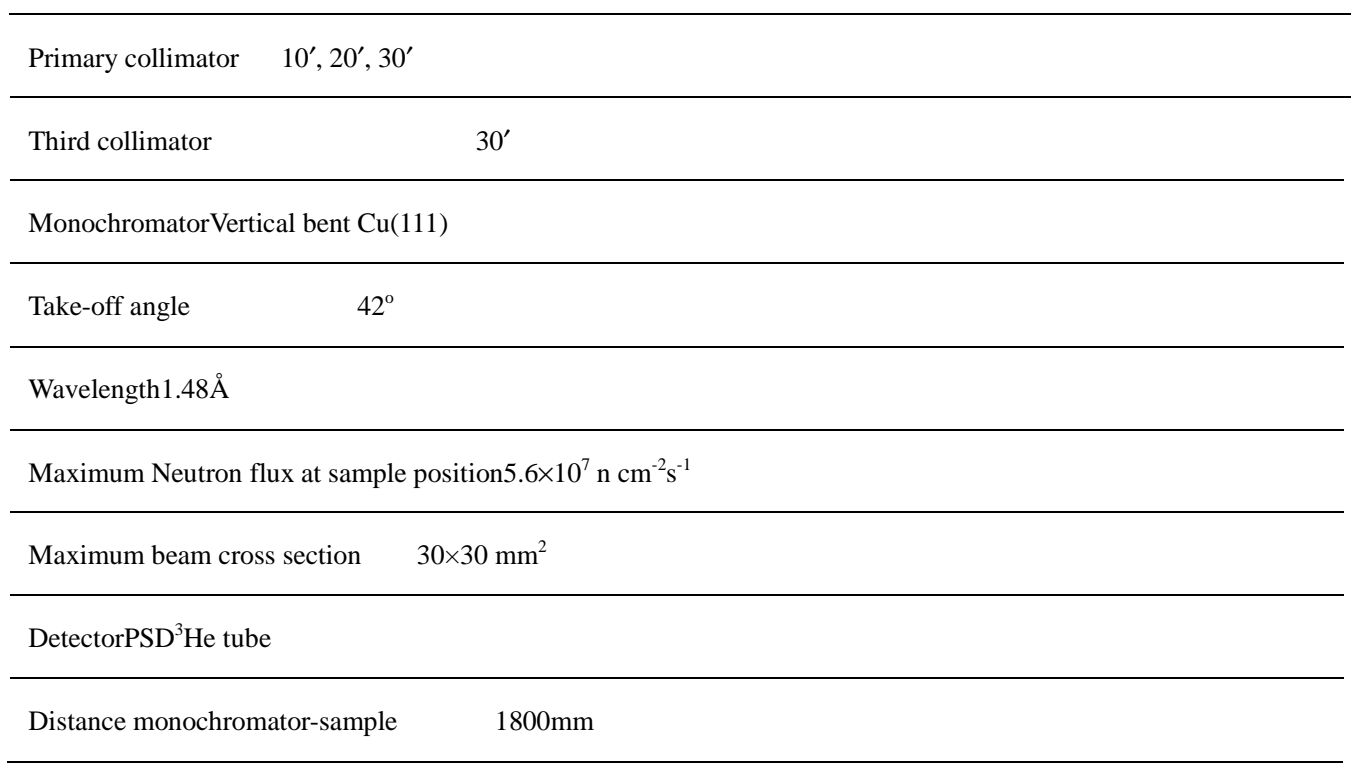




\section{Experimental measurements}

\subsection{Diffraction pattern measurement for sample $\mathrm{TiO}_{2}$}

The diffraction pattern of a cylindrical $\mathrm{TiO}_{2}$ sample with height $20 \mathrm{~mm}$ and diameter $10 \mathrm{~mm}$ was measured with $\alpha 1=30$ 'to calibrate this instrument. The sample was mounted on a thin $\mathrm{Al}$ rod wrapped by a $\mathrm{Cd}$ foil and inserted into the center of the goniometer. The neutron beam cross section is $20 \mathrm{~mm}$ (width) $\times 30 \mathrm{~mm}$ (height). A $2 \theta$-scan in the range of $25^{\circ}$ to $72^{\circ}$ was carried out at a step of $0.1^{\circ}$.The Rietveld profile technique was used to analyse the data with the program Fullprof, using the known structure of $\mathrm{TiO}_{2}$.

Figure 4 shows the measured and calculated diffraction patterns of the $\mathrm{TiO}_{2}$ sample. As can be seen, a good fit between them was achieved. The actual neutron wavelength $\lambda$ and the corresponding take-off angle $2 \theta_{M}$ thus obtained, are listed in Table 2.The real resolution curve of the instrument derived from the fit is plotted in Fig.5, together with the designed resolution curve. Good agreement between them is also found.

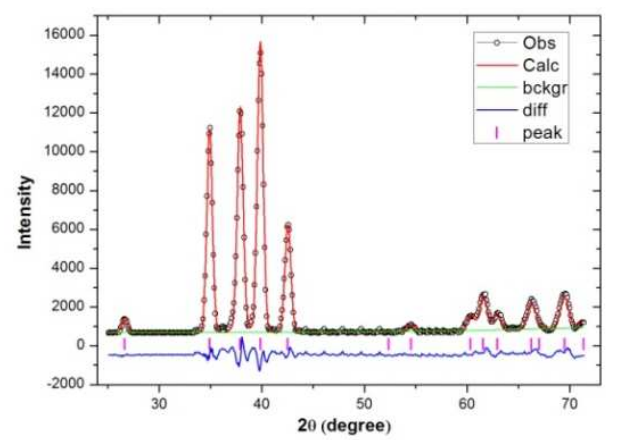

Figure 4 Neutrondiffraction pattern of $\mathrm{TiO}_{2}$ sample

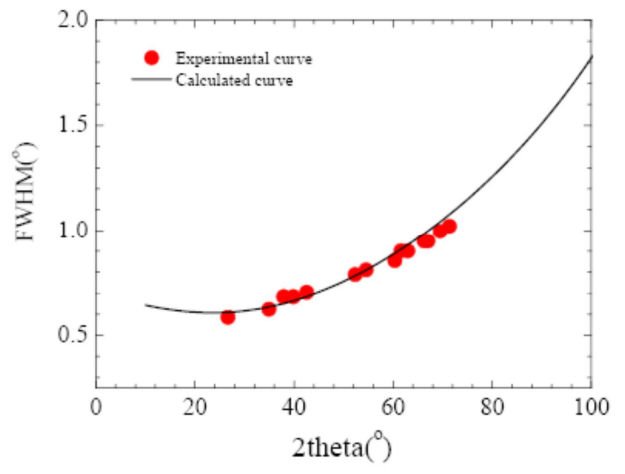

Figure 5Resolution curve of the texture diffractometer at CARR

\subsection{Neutron intensity measurement}

The gold foil activation method was adopted to measure the neutron flux at the sample position. A 
circular gold foil with diameter $19 \mathrm{~mm}$ was fixed at the center of the sample table, and totally bathed in the neutron beam for about 6 hours at the reactor power of 10MW. Through measuring the actively of ${ }^{198} \mathrm{Au}$ produced by the reaction of ${ }^{197} \mathrm{Au}(\mathrm{n}, \gamma){ }^{198} \mathrm{Au}$, the neutron flux was obtained. Corresponding to a reactor power of $60 \mathrm{MW}$, it is $5.6 * 10^{7} \mathrm{n} \mathrm{cm}^{-2} \mathrm{~s}^{-1}$ at the sample position, and is also listed in Table1.

\subsection{Pole figure measurements for warm-rolledZircaloy-4 plate}

The pole figures of a round-robin sample of warm-rolled Zircaloy-4 were measured to test the performance of this texture diffractometer. The texture measurements for this sample have also been carried out at the neutron texture diffractometers of High-Pressure-Preferred Orientation (HIPPO) at LANSCE and Kowari at ANSTO. A cubic sample of $12 \times 12 \times 12 \mathrm{~mm}^{3}$ was prepared by spark cutting a warm-rolled Zircaloy-4 plate into small squares, and then gluing them together along the same rolling direction. The size of incident neutron beam was set as $25 \times 25 \mathrm{~mm}^{2}$ by the variable slit to ensure the sample bathing in the neutron beam.

The (10-10), (0002), (10-11) and (11-20) pole figures were each measured at the reactor power of 10 MW. The time taken for each pole figure was about 4 hours. The raw data were transferred into LaboTex format, then used for quantitative ODF analysis. Shown in Figure 6 are the (0001), (10-10) and (11-20) pole figures, recalculated from the obtained ODF together with those measured at HIPPO and Kowari. Good agreement is found both qualitatively and quantitatively between the different instruments ${ }^{[16-17]}$.The Kearns factors and texture indices were also calculated, and the results are shown in Table 2.All Kearns factors obtained on different instruments are within an uncertainty of \pm 0.02 , which is lower than typical differences usually found for different batches, or between the start and end of pressure tubes ${ }^{[17-18]}$.

Table 2Kearns factors and texture index for the Zircaloy-4 specimens

Instrument TransverseNormalRollingSumText index

CARR-TD0.3520.5270.1180.9972.02

HIPPO0.3590.5430.0960.9982.15

Kowari0.3570.5510.0900.9982.32 


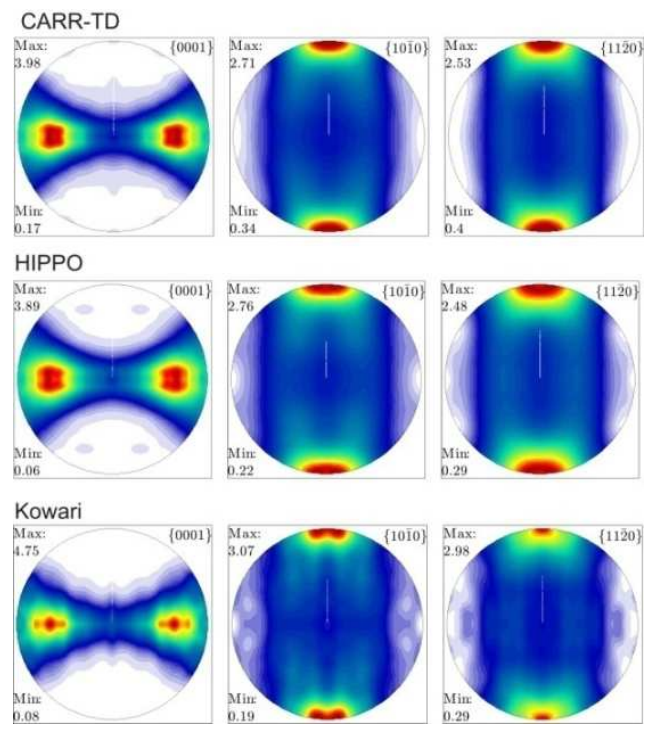

Figure6(0001), (10-10) and (11-20) pole figures recalculated from the ODFof warm-rolled Zircaloy-4 sample

\section{Summary}

The newly constructed neutron diffractometer at CARR is the first instrument in China for texture pole figure measurement by neutrons. With relatively high neutron intensity and performance, it has been proved to be efficient and suitable for pole figure measurements for simple structure materials in the present stage. Up to now, a number of pole figures with satisfying quality have been obtained on this instrument for different research projects. A further improvement in its performance will be made in the near future by replacing the single ${ }^{3} \mathrm{He}$ detector with a two dimensional PSD.

The NTD at CARR has been opened to the user community. Users are welcome to apply to use this instrument for their research projects independently or in cooperation with us.

\section{Acknowledgements}

The author Meijuan LI thanks Prof. Baisheng Zhang for helpful discussion.This works supported by the National Nature Science Foundation of China (No. 11105231), International Atomic Energy Agency- TC program (No.CPR0012) and National Nature Science Foundation of China (No. 11205248).

\section{References}

[1]Bunge HJ, Vi International School On Neutron Physics, Texture analysis by neutron diffraction, 1990, 8-18 October

[2]Wenk H R, HoutteP V, Reports On Progress In Physics, 2004, 67:1367-1428

[3]Brokmeier HG, PhysicaB, 1997, 234- 236: 977-979

[4]Bunge H J, Textures and Microstructures, 1989, 10: 265-307

[5]Brokmeier HG, Physica B,1997, 234-236:1144-1145 
[6]CHEN Dong-Feng, LIU Yun-Tao,WU Mei-Mei, A Progress Report. Neutron News. 2009, 20 (2): 32-33

[7]CHEN Dong-Feng, LIU Yun-Tao,GOU Cheng,Physica B, 2006, 385 - 386: 966 - 967

[8]HEWAT A W, Nuclear Instruments And Methods, 1975, 127: 361-370

[9] WenkH R, LutterottiL, Vogel S, Nuclear Instruments And Methods in Physics Research A, 2003, 515: 575-588

[10]Caglioti G, Paoletti A,Ricci F P, Nuclear Instruments and Methods, 1958, 3(4): 223-228

[11] Caglioti G, Paoletti A, Ricci F P, Nuclear Instruments and Methods, 1960, 9(2): 195-198

[12] CagliotiG, Ricci F P, Nuclear Instruments and Methods, 1962, 15 (2):155-163

[13] LIU Xiao-Long, Design and key technologystudy of the Neutron Texture Diffractometer at CARR (Ph.D. Thesis). Beijing: China Institute of Atomic Energy,2012 (in Chinese)

[14] LI Mei-Juan, LIU Yun-Tao,LIU Xiao-Long, et al. Materials Science Forum, 2014, 777: 71-77

[15]LIU Xiao-Long, CHEN Dong-Feng, LI Mei-juan, et al. Atomic Energy Science and Technology, 2013,47 (8): $1435-1439$

[16]WenkHR, LutterottiL, Vogel S, Nuclear Instruments and Methods in Physics Research A, 2003, 515: 575-588

[17]Santisteban JR, Malamud F, Vizcaino P, et al. Proposal for a Round Robin on neutron diffraction determination of texture of

Zr-based alloysCoordinated by IAEA within the CRP, 2013

[18]BuioliC, Proc $39^{\text {th }}$ Meeting of Argentina Nuclear Technology Association-AATN, 2012 